\title{
Central versus Local Quality Efforts: The Need for Both
}

\author{
Aleece Caron, $\mathrm{PhD}$ and Peter Pronovost, $\mathrm{MD}, \mathrm{PhD}$
}

Healthcare is in need of improvement. It harms too often, costs too much, learns and improves too slowly, and burns out its workforce. Large healthcare systems (HCS) have an important role in influencing the quality and value of care. Still, as systems that, in most cases, have grown and emerged rapidly in the last 20 years, few have organizational structures to support and foster the last aim, creating the conditions for the healthcare workforce to find joy and meaning in their work. HCS struggle to develop quality improvement (QI) because they are diverse and dynamic in composition, size, resources, culture and social structures, and needs. This diversity may drive forces for change or may undermine QI efforts. Clinical teams often rely on local QI efforts to improve care at the delivery site. At the same time, managers and executives focus on a centralized, system-wide approach, generally focused on externally reported metrics. We propose that a hybrid of the 2 most popular healthcare QI approaches, local QI and centralized QI, might be the best method for achieving and sustaining quality care across a wide variety of conditions. ( $\mathrm{J}$ Am Board Fam Med 2021;34:1038-1041.)

Keywords: Delivery of Health Care, Leadership, Quality Improvement, Quality of Health Care, Systems

Healthcare is in need of improvement. It harms too often, costs too much, learns and improves too slowly, and burns out its workforce. As healthcare systems (HCS) are implementing programs and processes that: improve patient experience, enhance the health of populations, reduce costs of care, and improve the work-life and well-being of clinicians. These combined efforts are part of the Quadruple Aim. ${ }^{1}$ These improvement efforts combine local efforts, focusing on local opportunities, and centralized efforts across a health system, largely focusing on measures used in pay for quality programs and reported publicly. While it may seem common sense that local and centralized improvement efforts should align, they are disconnected,

This article was externally peer reviewed.

Submitted 9 January 2021; revised 19 March 2021; accepted 15 April 2021.

From the Case Western Reserve University, Case Western Reserve University School Of Medicine, Cleveland, OH (AC, PP); The Population Health Research Institute at the MetroHealth System, Cleveland, OH (AC); University Hospitals, Cleveland, OH (PP).

Funding: Internally funded.

Conflict of interest: None.

Contributions: AC is the Co-Director of the Population Health Research Institute at the MetroHealth System and an Associate Professor of Medicine at Case Western Reserve University. She serves as the Project Director of HRSA funded Primary Care Champions grant that offers mentored practice transformation training to primary care providers, physician assistants (PA), and $\mathrm{PA}$ students in reducing the effectiveness of both and often burdening clinicians with confusing and conflicting goals and chaotic projects. In this essay, we present an approach to harmonize local and centralized improvement efforts.

While large HCS have an important role in influencing the quality and value of care, they usually have fragmented productivity-focused processes that result in increased work to achieve desired improvements. Providers and provider teams are often not included in an improvement effort but are responsible for achieving improved results. ${ }^{2}$ This barrier contributes to high burnout and increased attrition measured among primary care providers. A robust quality improvement (QI) culture that emphasizes

urban underserved clinics. AC has trained over 50 participants from primary care clinics and has expanded the training beyond Ohio. As Senior Practice Consultant for Better Health Partnership, she mentored and facilitated primary care practice transformation projects throughout our geographic region. Her work in this area was recognized as a best practice by the National Committee for Quality Assurance. PP is an internationally acclaimed leader in patient safety who has distinguished himself nationally and internationally with his ground-breaking work around saving lives, improving patient safety, and improving both the quality and value of healthcare. His life-saving clinical practices have yielded dramatic improvements in hospitals across the United States and around the world.

Corresponding author: Aleece Caron, PhD, 2500 MetroHealth Drive, Cleveland, OH 44109, Telephone: 216-210-3995 (E-mail: aleece.caron@case.edu). 
teamwork, individual growth, and innovation is the needed infrastructure to sustain the realization of Quadruple Aim. ${ }^{3}$

Achieving and sustaining a culture of QI in large HCS requires a shared understanding across different levels of the hospital of the improvement priorities. ${ }^{3}$ HCS leadership often prioritizes QI goals based on financial reimbursement or metrics that are publicly reported. Individual clinics, which provide direct care to patients, are often not consulted regarding their role in achieving QI metrics or about whether those metrics are relevant to the care they deliver. Few clinical practices are engaged in how best to address institutional QI goals.

We propose that a hybrid, local QI, and centralized QI, might be the best method for achieving and sustaining quality care across a wide variety of conditions. ${ }^{4,5}$ This hybrid aligns awareness, prioritization, and value of efforts in a HCS between system leadership at large and local clinical units. Healthcare delivery systems are becoming increasingly consolidated in the United States, and large HCS are buying individual practices. ${ }^{6}$ These practices will need guidance, training, and resources from leadership about how to merge their QI culture with centralized QI efforts common in larger HCS. To successfully implement system-level interventions to fit within the context of local practice, leaders need to work with frontline staff to co-design and adapt the intervention to work with the structure and processes of the practice. This requires system leaders to have communication channels to address the technical and adaptive components of change to achieve wide-scale adoption and structures to connect system and local leaders to support the co-creation of goals, peer learning, and accountability. $^{7,8}$

Local efforts often struggle to achieve success because they have limited time and resources to work on QI initiatives, may lack the necessary expertise, or may not know the evidence to support the intervention. System leadership is often unaware of these efforts and their potential value and rarely provides time and resources for staff to excel in local QI projects. However, evidence suggests that local efforts are effective at improving care quality, provider satisfaction, provider retention, and patient experience without affecting clinical productivity. ${ }^{9}$ Lack of central leadership support is may be in part due to the fallacy that staff who participate in local efforts will negatively affect clinical productivity or insignificantly improve care value. ${ }^{10}$

\section{Case Example}

The MetroHealth System (MHS), Cuyahoga County's safety-net hospital, located on the west side of Cleveland 8 miles from Case Western Reserve University (CWRU), is an integrated health system with an acute care hospital housing the area's only burn center and a level 1 adult trauma center. The emergency department is among the busiest in the country ( $>275$ visits per day); Metro Life Flight air ambulance service is internationally recognized. In 1999, MHS was the first public safety-net hospital system in the United States to install an electronic health record (EHR) for clinical care and has been recognized by several national organizations for its early adoption and sustained value of its EHR. Over $40 \%$ of MHS's socioeconomically diverse patient population is enrolled in Epic's MyChart personal health record. As a system, MHS provided 417,000 outpatient visits to uninsured and Medicaid patients in 2019.

MHS allocated time for primary care providers to receive training in QI methods, addressing social determinants of health, developing business cases, and providing patient-centered care, with the expectation that they would lead a QI effort at their clinical sites of care. Fourteen providers took part in the initial training. Primary care clinical quality metrics, such as vaccinations, improved. From 6 months before program participation compared with the 6 since months of program participation, Pneumonia vaccination rates improved by $5 \%$, influenza vaccinations improved by $4 \%$, and tetanus by $8 \%$. In addition, patient experience scores, as measured by how likely patients were to recommend their provider, improved anywhere from 5\% to $20 \%$. After participating in the program, providers indicated they were more likely to remain at the institution, were more satisfied with their jobs, and were less likely to experience burnout. There was less than a $0.5 \%$ decrease in overall clinical productivity as measured by work relative value units, equaling a total average annual program cost in lost clinical revenue of $\sim \$ 12,000$ for 14 participants ( $<\$ 1,000$ per participant). While we did not look at efficiency, as mentioned earlier, we saw improvement in quality outcomes. In addition, academic 
productivity gains have improved with no further loss of clinical productivity. One of the scholars was promoted to Program Director in Family Medicine, one coedited a book on health disparities, one led the successful system effort for National Committee for Quality Assurance (NCQA) Patient-Centered Medical Home Level 3 recognition, and one was promoted to the Institutional Leader in data-driven quality improvement. The total number of peer-reviewed publications produced by the faculty scholars increased from zero in the 2-year period before enrollment to 9 in the 3 years of the program. The total number of national meeting presentations increased from 11 to 30 . If healthcare providers have time to participate in workforce development opportunities, they can apply quality improvement methods to reducing errors, improving value, address system issues, and addressing social determinants of health. In our system, this pilot program demonstrated value to the institution, and leadership continues to support it and expand eligibility different healthcare team members. In some cases, it uses the program as a recruiting incentive.

Ideally, local and centralized efforts should be aligned, in large part because they should address the same goals. However, centralized efforts are often focused on meeting or exceeding external benchmarks, and local efforts address everyday pain points that may represent a significant risk to patients and are often not communicated to or prioritized by organizational leadership. The goal is to provide standardized, high-quality care for all patients with the quality characteristic that needs improvement. QI efforts often do not achieve the desired outcomes for various reasons. ${ }^{11}$ Yet, a common reason is they often lack a robust operating model that addresses the technical and adaptive components of change. ${ }^{12}$ Leadership fails to include local teams in designing the intervention and fails to recognize negative impacts of the new process on the staff and other work done by the local teams. It is important for leaders to understand how to drive behavior change. Behavior can change by regulation or fiat, by incentives, or by creating peer networks to establish social norms is a critical component to success. ${ }^{13}$ These peer learning communities are potent yet underutilized tools in system-level change. ${ }^{14}$

Long-term healthcare leaders must strongly promote sharing, aligning, and integrating the changing larger HCS priorities with the local HCS units for success and adaptability. ${ }^{15}$ HCS with organizational structures and processes that balance HCS QI priorities with local QI innovation and solutions will be more likely to obtain staff buy-in, support teamwork, and acknowledge that frontline staff is uniquely qualified to fit organizational QI interventions to their local context. Unsupported local QI efforts could demoralize front-line teams and negatively impact the quality metrics that hospital leadership supports. Moreover, the HCS is less likely to benefit from best practice solutions that can come from local efforts and be disseminated at large.

System leadership needs to hold local QI efforts accountable for their outcomes and review these local efforts with the same rigor as the centralized QI efforts. Deep system learning requires relationships, collaboration, and shared values for the work overtime. It is critical to align and support local, and systems-level efforts as both are important and need to be supported. Leaders need to create mechanisms to support the dissemination of best practices and engage local experts to support centralized efforts to create and maintain a culture of change. Large HCS must share priorities, knowledge, leadership, and credit at local levels if they are to be places to grow the culture of partnership and innovation needed now and in the future to reach the Quadruple Aim.

To see this article online, please go to: http://jabfm.org/content/ 34/5/1038.full.

\section{References}

1. Bodenheimer T, Sinsky C. From triple to quadruple aim: care of the patient requires care of the provider. Ann Fam Med 2014;12:573-6.

2. Kravet SJ, Bailey J, Demski R, Pronovost P. Establishing an ambulatory medicine quality and safety oversight structure. Acad Med 2016;91:962-6.

3. Souza JP, Alves JM. Lean-integrated management system: A model for sustainability improvement. J Clean Prod 2018;172:2667-82.

4. Robert G, Cornwell J, Locock L, Purushotham A, Sturmey G, Gager M. Patients and staff as codesigners of healthcare services. BMJ 2015;

5. Ward ME, De Brún A, Beirne D, et al. Using codesign to develop a collective leadership intervention for healthcare teams to improve safety culture. IJERPH 2018;15:1182.

6. Hughes R. Patient Safety and Quality: An EvidenceBased Handbook for Nurses. Available from: https:// www.researchgate.net/publication/268043663_Patient_ 
Safety_and_Quality_An_Evidence-Based_Handbook_ for_Nurses. Published 2008. Accessed April 13, 2021.

7. Heifetz R, Grashow A, Linsky M. The practice of adaptive leadership: Tools and tactics for changing your organization and the world. Harvard Business Press, 2009.

8. Pronovost PJ, Marsteller JA. Creating a fractalbased quality management infrastructure. J Health Organ Manag 2014;28:576-86.

9. Bloom N, Brynjolfsson E, Foster L, et al. What drives differences in management practices? Am Econ Rev 2019;109:1648-83.

10. Johnson SG, Zhang J, Keil F. Win-Win Denial: The Psychological Underpinnings of Zero-Sum Thinking 2020.

11. White M, Wells JSG, Butterworth T. The impact of a large-scale quality improvement programme on work engagement: preliminary results from a national cross-sectional-survey of the 'Productive Ward.' Int J Nurs Stud 2014;51:1634-43.

12. Pronovost PJ, Urwin JW, Beck E, et al. Making a dent in the trillion-dollar problem: toward zero defects. N Engl J Med Catalyst 2021;2: 542-67.

13. Christianson J, Carlin C, Warrick L. The dynamics of community healthcare consolidation: acquisition of physician practices. Milbank Q 2014;92.

14. Dixon-Woods M, Bosk C, Aveling E, Coeschel C, Pronovost P. Explaining Michigan: developing an ex post theory of a quality improvement program. Milbank Q 2011;89:167-205.

15. Rogers EM, Shoemaker FF. Communication of Innovations; A Cross-Cultural Approach. 2d ed. New York: Free Press. 1971. 